\title{
The True Human Cost of the Novel Coronavirus 2019 (COVID-19) Pandemic
}

\author{
Rohan Chaubal',2,3(1) Sudeep Gupta2,3,4이 \\ ${ }^{1}$ Department of Surgical Oncology, Tata Memorial Centre, Mumbai, \\ Maharashtra, India \\ ${ }^{2}$ Hypoxia and Clinical Genomics Laboratory, ACTREC-TMC, Mumbai, \\ Maharashtra, India \\ ${ }^{3}$ Homi Bhabha National Institute, Mumbai, Maharashtra, India \\ ${ }^{4}$ Department of Medical Oncology, Tata Memorial Centre, Mumbai, \\ Maharashtra, India
}

Ind J Med Paediatr Oncol 2022;43:378-381.

As of 6th June 2021, 3:00 pM, there were 174,893,444 worldwide reported cases of the novel coronavirus disease 2019 (COVID-19), with 3,774,138 deaths attributed to this pandemic. ${ }^{1,2}$ Infection fatality rate (IFR) i.e., the fraction of deaths reported from all laboratory-confirmed cases for the disease, for COVID-19 has thus far been estimated in a broad range from 0.5 to $1.0 \%{ }^{3,4}$ The IFR for any disease is influenced by the accuracy of two metrics: the death reporting mechanism and its attribution to the disease, and testing every possible individual who may have contracted the disease.

Both COVID-19 death reporting and testing are contentious issues, with many countries suspected to be underreporting deaths because of errors of omission and/or commission. ${ }^{5}$ This is because, in almost all countries, deaths outside the hospital system are inaccurately reported. Many individuals who die from COVID-19-like symptoms do not get a laboratory confirmatory test. Moreover, asymptomatic individuals constitute a large proportion of COVID-19 cases, ${ }^{6}$ accounting for as much as 40 to $45 \%$ of total SARS-CoV2 infections. ${ }^{7,8}$ Many countries with low resources or with limited testing availability do not even test mildly symptomatic individuals due to scarce availability of RT-PCR testing, ${ }^{9,10}$ leading to weak attributable death reporting systems. Thus, estimating IFR accurately is extremely challenging.

So just how many people have died of COVID-19 is a question with huge ramifications for public health policymaking. ${ }^{11,12}$ Unfortunately, given the times we live in, this is also a question fraught with political overtones, whose answer depending on whom you ask, range from a benign
Address for correspondence Sudeep Gupta, MD, DM, Department of Medical Oncology, Tata Memorial Centre, Room 1109, 11th Floor, HBB, Mumbai-400012, Maharashtra, India (e-mail: sudeepgupta04@yahoo.com).

infection no deadlier than the common seasonal influenza to the deadliest disease since the black plague. As always, the truth lies somewhere in between. Recent newspaper and magazine articles in The New York Times (NYT) $)^{13}$ and The Economist ${ }^{14}$ estimating excess COVID-19 deaths for the period March 2020 to May 2021 have received widespread media coverage. However, both are deficient in scientific rigor. The NYT analysis is a simple linear regression model of varying ranges of COVID-19 mortality as extrapolated from hypothesized rates of IFR, one conservative $(496,000$ excess deaths in India), and the other, an extreme (4.5 million excess deaths in India), with a mid-point spread estimate (2.1 million excess deaths). ${ }^{15}$ The Economist analysis, in contrast, while using the same method as that of NYT at its core, also uses certain assumptions to fit its model, using a machine learning methodology, ${ }^{16}$ many of which simply do not hold true in the real world. The analysis suggests a point estimate spread of 7 million (conservative scenario) to 13 million (worst case scenario) excess COVID-19 deaths worldwide, 1 million of these from India alone (worst case scenario). ${ }^{17}$

One underreported analysis that has received lesser coverage but is perhaps one of the most credible, is by the Institute for Health Metrics and Evaluation (IHME), at the University of Washington. ${ }^{18}$ IHME has perfected and implemented a long-standing methodology for measuring the burden of diseases on a global scale since 1990 through its Global Burden of Disease (GBD) study. ${ }^{19}$ The GBD estimates worldwide deaths annually due to aging, communicable
DOI https://doi.org/ 10.1055/s-0041-1740320. ISSN 0971-5851.
(C) 2021. Indian Society of Medical and Paediatric Oncology. All rights reserved.

This is an open access article published by Thieme under the terms of the Creative Commons Attribution-NonDerivative-NonCommercial-License, permitting copying and reproduction so long as the original work is given appropriate credit. Contents may not be used for commercial purposes, or adapted, remixed, transformed or built upon. (https://creativecommons.org/ licenses/by-nc-nd/4.0/)

Thieme Medical and Scientific Publishers Pvt. Ltd., A-12, 2nd Floor, Sector 2, Noida-201301 UP, India 
diseases, non-communicable diseases (including psychiatric disorders), and accidental deaths (traffic, personal injuries, occupational etc.). This is thus far the most comprehensive modeling of all-cause mortality worldwide.

In its special COVID-19 mortality analysis, IHME has used the data from its annual GBD modeling methodology from the period of March 1, 2020, to May 13, 2021, to estimate and predict the global burden of deaths specifically due to COVID-19 during this period. Their methodology is broadly as follows:

1) Using their GBD models, they first estimated the expected all-cause mortality in the period March 1,
2020, to May 13, 2021, based on pre-pandemic deaths, i.e., how many people would have died in this period if there was no COVID-19 pandemic.

2) They then collected the total deaths that were actually reported by countries during this period from allcause mortality.

3) This was subtracted from the total expected deaths in this period in the absence of the COVID-19 pandemic.

4) The excess mortality thus arrived at was further modeled to identify variables including a lag in death reporting, decrease in mortality due to COVID-19, and increase in mortality directly and indirectly due to COVID-19.
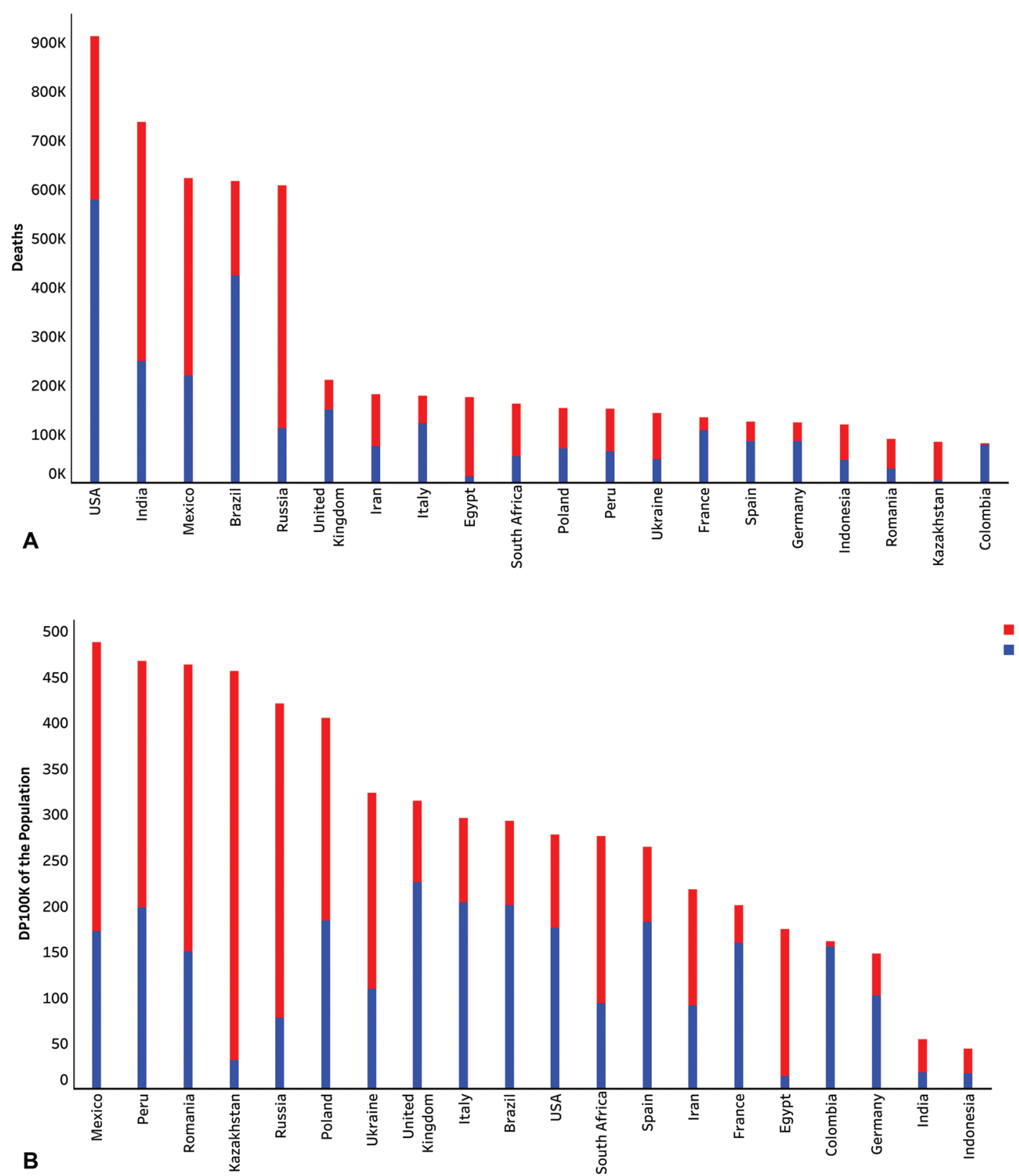

Fig. 1 (A) Blue bar indicates absolute number of reported deaths due to COVID-19. Red bar represents the imputed excess deaths due to COVID-19 in the studied time period. Red and blue bars together constitute cumulative deaths due to COVID-19 in the studied time period for that country (X-axis). (B) Blue bar indicates reported deaths per 100,000 of the population (DP100K) due to COVID-19. Red bar represents the imputed deaths per 100,000 of the population (DP100K) due to COVID-19 in the studied time period. Red and blue bars together constitute cumulative deaths per 100,000 of the population (DP100K) due to COVID-19 in the studied time period for that country (X-axis). DP100K: Deaths per 100,000 population. 
Their final model thus was able to predict the following different causes of mortality

1) all deaths directly on account of a laboratory-confirmed COVID-19 infection

2) increase in mortality due to

i. life-saving health care (including disease screening) being delayed or deferred during the pandemic.

ii. mental health disorders (depression) due to COVID-19 isolation/lockdowns, loss of earnings, and resulting opioid use.

iii. excessive alcoholism.

3) reduction in mortality due to:

i. decreased mobility associated with social distancing mandates

ii. reduced transmission of other viruses (influenza, respiratory syncytial virus, and measles)

iii. individuals at immediate risk of dying from chronic conditions, such as cardiovascular disease and chronic respiratory disease, dying instead from COVID-19.

The IHME reports the largest number of cumulative deaths worldwide accounting for underreported deaths are from the USA (912,345), India (736,811), Mexico (621,962), Brazil
(616,914), and Russia $(607,589)$ ( - Fig. 1A). This is not surprising because these countries have had the largest epidemics to date. Interestingly, but not unexpectedly, some countries with relatively smaller epidemics saw a large increase in the death rate when accounting for unreported deaths, most of these in South America, Central Asia, and Eastern Europe (-Fig. 1B). We divided the excess death count for each country (as estimated by the IHME), with the reported death counts for that country. The excess death ratio thus arrived at revealed that 12 countries had undercounted their deaths by at least as much as their total reported deaths, 6 countries had undercounted by close to half the total reported deaths, while 2 countries had undercounted by a low proportion ( - Table 1 ). The IHME estimates the global COVID-19 death rate is 91.7 per 100,000 . Vietnam has the lowest total COVID-19 death rate at 0.1 per 100,000 , while a staggering 15 countries have total COVID-19 death rates per 100,000 (DP100K) higher than 200

(-Table 1, - Fig. 1B). These findings, besides shedding light on the true human cost associated with this pandemic, also reveal an important detail: some countries have done exceedingly well at preventing deaths, while for unknown reasons that we can only speculate upon at this point of time, other countries have been less nimble. ${ }^{12}$ Clearly, only data based public health interventions will help formulate policies that can tackle this pandemic to prevent further loss of life.

Table 1 Country-wise reported and excess deaths as per IHME estimates (columns 1-4) and estimated total deaths including per 100 thousand population as estimated by us based on data from the IHME analysis (columns 5-7)

\begin{tabular}{|c|c|c|c|c|c|c|}
\hline Country & $\begin{array}{l}\text { Reported COVID-19 } \\
\text { deaths }\end{array}$ & $\begin{array}{l}\text { Excess } \\
\text { deaths }\end{array}$ & $\begin{array}{l}\text { Total COVID-19 } \\
\text { deaths }\end{array}$ & $\begin{array}{l}\text { Reported COVID-19 } \\
\text { deaths per } 100 \mathrm{~K}\end{array}$ & $\begin{array}{l}\text { Total COVID-19 } \\
\text { deaths per 100K }\end{array}$ & $\begin{array}{l}\text { Increase } \\
\text { in deaths } \\
\text { per } 100 \mathrm{~K}\end{array}$ \\
\hline Kazakhstan & 5,810 & 78,643 & 84,453 & 31.39 & 456.16 & 424.77 \\
\hline Russia & 111,909 & 495,680 & 607,589 & 77.52 & 420.85 & 343.33 \\
\hline Mexico & 219,372 & 402,590 & 621,962 & 171.96 & 487.53 & 315.57 \\
\hline Romania & 29,020 & 60,599 & 89,619 & 149.93 & 463 & 313.07 \\
\hline Peru & 64,511 & 87,428 & 151,939 & 198.44 & 467.36 & 268.92 \\
\hline Poland & 69,954 & 83,672 & 153,626 & 184.24 & 404.59 & 220.35 \\
\hline Ukraine & 48,393 & 95,022 & 143,415 & 109.03 & 323.12 & 214.09 \\
\hline South Africa & 54,746 & 106,758 & 161,504 & 93.49 & 275.81 & 182.32 \\
\hline Egypt & 13,970 & 161,620 & 175,590 & 13.92 & 174.92 & 161 \\
\hline Iran & 75,547 & 104,940 & 180,487 & 91.12 & 217.69 & 126.57 \\
\hline USA & 578,555 & 333,790 & 912,345 & 176.27 & 277.96 & 101.69 \\
\hline Brazil & 423,307 & 193,607 & 616,914 & 200.58 & 292.31 & 91.73 \\
\hline Italy & 122,851 & 55,293 & 178,144 & 203.75 & 295.45 & 91.7 \\
\hline United Kingdom & 150,815 & 59,261 & 210,076 & 225.66 & 314.33 & 88.67 \\
\hline Spain & 85,822 & 38,627 & 124,449 & 182.31 & 264.36 & 82.05 \\
\hline Germany & 84,807 & 38,170 & 122,977 & 102.02 & 147.93 & 45.91 \\
\hline France & 106,874 & 27,526 & 134,400 & 159.38 & 200.42 & 41.04 \\
\hline India & 248,016 & 488,795 & 736,811 & 18.16 & 53.93 & 35.77 \\
\hline Indonesia & 47,150 & 71,646 & 118,796 & 17.43 & 43.9 & 26.47 \\
\hline Colombia & 78,216 & 2,752 & 80,968 & 155.38 & 160.85 & 5.47 \\
\hline
\end{tabular}




\section{Statement of Integrity}

The manuscript has been read and approved by all authors and the requirements for authorship have been met, and that each author believes that the manuscript represents honest work.

\section{Funding}

This study report was self-funded. R.C. acknowledges the funding support of the PhD Health Sciences fellowship from Tata Memorial Hospital, Mumbai, and The Homi Bhabha National Institute, Mumbai, and from the Virtual National Cancer Institute (VNCI) Breast Cancer grant, received by S.G. from the Department of Biotechnology, GOI. The funders had no role to play in this report.

\section{Conflict of Interest}

None declared.

\section{References}

1 GitHub repository for Coronavirus COVID-19 global cases by the Center for Systems Science and Engineering (CSSE) at Johns Hopkins. Updated 09 February 2021. Accessed January 22, 2021 at: https://github.com/CSSEGISandData/COVID-19

2 Dong E, Du H, Gardner L. An interactive web-based dashboard to track COVID-19 in real time. Lancet Infect Dis 2020;20(05): 533-534

3 Pastor-Barriuso R, Pérez-Gómez B, Hernán MA, et al; ENE-COVID Study Group. Infection fatality risk for SARS-CoV-2 in community dwelling population of Spain: nationwide seroepidemiological study. BMJ 2020;371:m4509. Doi: 10.1136/bmj.m4509

4 Perez-Saez J, Lauer SA, Kaiser L, et al; Serocov-POP Study Group. Serology-informed estimates of SARS-CoV-2 infection fatality risk in Geneva, Switzerland. Lancet Infect Dis 2021;21(04): e69-e70

5 Burn-Murdoch JRVaGC. Global coronavirus death toll could be 60\% higher than reported. Financial Times. April 26, 2020. Accessed April 26, 2020 at: https://www.ft.com/content/ 6bd88b7d-3386-4543-b2e9-0d5c6fac846c

6 Ward H, Atchison C, Whitaker M, et al. SARS-CoV-2 antibody prevalence in England following the first peak of the pandemic. Nat Commun 2021;12(01):905. Doi: 10.1038/s41467-02121237-w
7 Oran DP, Topol EJ. Prevalence of asymptomatic SARS-CoV-2 infection. Ann Intern Med 2021;174(02):286-287

8 Almadhi MA, Abdulrahman A, Sharaf SA, et al. The high prevalence of asymptomatic SARS-CoV-2 infection reveals the silent spread of COVID-19. Int J Infect Dis 2021;105:656-661

9 Chakraborty C, Ranjan Sharma A, Bhattacharya M, Lee SS, Agoramoorthy G. COVID-19 vaccine: challenges in developing countries and India's initiatives. Infez Med 2021;29(01):165-166

10 Anjum FR, Anam S, Rahman SU. Novel Coronavirus disease 2019 (COVID-19): new challenges and new responsibilities in developing countries. Hum Vaccin Immunother 2020;16(10):2370-2372

11 Xie Y, Bowe B, Maddukuri G, Al-Aly Z. Comparative evaluation of clinical manifestations and risk of death in patients admitted to hospital with COVID-19 and seasonal influenza: cohort study. BMJ 2020;371:m4677. Doi: 10.1136/bmj.m4677

12 Chaubal R, Kannan S, Khattry N, Gupta S. Worldwide case fatality ratio of COVID-19 over time. Preprint 2021

13 Jin Wu AM Josh Katz, Elian Peltier and Karan Deep Singh. The Pandemic's Hidden Toll: Half a Million Deaths. The New York Times. 09-02-2021. Accessed June 13, 2021 at: https://www.nytimes.com/ interactive/2020/04/21/world/coronavirus-missing-deaths.html

14 Team TEDM. Counting the dead- Modelling covid-19's death toll: There have been $7 m-13 m$ excess deaths worldwide during the pandemic. The Economist. 15 May 2021 ed2021.https://www. economist.com/briefing/2021/05/15/there-have-been-7m-13mexcess-deaths-worldwide-during-the-pandemic

15 Glanz LGaJ. Just How Big Could India's True Covid Toll Be? The New York Times. 25-05-2021. Accessed June 13, 2021 at: https://www. nytimes.com/interactive/2021/05/25/world/asia/india-covid-deathestimates.html

16 Team TEDM. Covid-19 death tallies- How we estimated the true death toll of the pandemic: Dealing with potential outcomes, known unknowns, and uncertainty. The Economist. 13-05-2021 ed: The Economist.

17 Team TEDM. More evidence emerges of India's true death toll from covid-19. The Economist. 12-06-2021 ed: The Economist; 2021

18 IHME. Estimation of total mortality due to COVID-19. 13-062021, 2021. Updated 13-05-2021. Accessed June 13, 2021 at: http://www.healthdata.org/special-analysis/estimation-excessmortality-due-covid-19-and-scalars-reported-covid-19-deaths

19 Diseases GBD, Injuries CGBD 2019 Diseases and Injuries Collaborators. Global burden of 369 diseases and injuries in 204 countries and territories, 1990-2019: a systematic analysis for the Global Burden of Disease Study 2019. Lancet 2020;396 (10258):1204-1222 